\title{
L'intégration des immigrants jamaïcains à Miami et Toronto
}

\section{Terry-Ann Jones}

Traducteur : Sabine Erbès-Seguin

\section{(2) OpenEdition \\ Journals}

\section{Édition électronique}

URL : https://journals.openedition.org/remi/4308

DOI : $10.4000 /$ remi.4308

ISSN : $1777-5418$

\section{Éditeur}

Université de Poitiers

\section{Édition imprimée}

Date de publication : 1 avril 2008

Pagination : 139-158

ISBN : 978-2-911-627-48-2

ISSN : 0765-0752

\section{Référence électronique}

Terry-Ann Jones, "L'intégration des immigrants jamaïcains à Miami et Toronto », Revue européenne des migrations internationales [En ligne], vol. 24 - $n^{\circ} 1$ | 2008, mis en ligne le 01 avril 2011, consulté le 14 avril 2022. URL : http://journals.openedition.org/remi/4308 ; DOI : https://doi.org/10.4000/remi.4308

Ce document a été généré automatiquement le 14 avril 2022.

(c) Université de Poitiers 


\title{
L'intégration des immigrants jamaïcains à Miami et Toronto
}

\author{
Terry-Ann Jones
}

Traduction : Sabine Erbès-Seguin

1 En dépit des similitudes entre les États-Unis et le Canada, il existe des différences subtiles, mais palpables, entre les deux pays. Alors que les États-Unis accueillent une population jamaïcaine beaucoup plus importante que celle du Canada - de respectivement 554000 et 122340 individus - les aires métropolitaines de Miami et de Toronto ont des populations jamaïcaines comparables, de respectivement 94691 et 93 315 personnes. Il existe cependant une différence de statut économique entre les deux populations, mesurée par les niveaux de revenus et d'éducation, tous deux plus élevés à Miami qu'à Toronto. Le présent travail étudie quelques-unes des raisons de ces divergences. Les politiques d'immigration, qui influent dans certains cas sur les possibilités d'emploi des immigrants et leur accès au marché du travail, ont une grande importance pour le potentiel de mobilité socio-économique ascendante.

2 L'insertion des immigrants dans la société d'accueil se fait par paliers, dont le premier, selon Portes et Rumbaut, renvoie à une acculturation, l'intégration étant le stade final du processus. Dans le passé, les modèles d'intégration se définissaient à partir de l'hypothèse d'une perte de la langue et de la culture des immigrants, et surtout de leurs enfants, et de leur substitution in fine par l'adoption de la langue et de la culture du « courant social et politique dominant " (Portes et Rumbaut, 1996 : 247-248). Ce modèle uniforme d'intégration a été largement rejeté (Portes et Zhou,1993; Portes et Rumbaut, 1996 ; Zhou, 1999; Rumbaut et Portes, 2001), et une approche alternative, celle de l'assimilation segmentée, est apparue afin de compenser quelques-unes des insuffisances du précédent modèle d'intégration. L'assimilation segmentée affirme la diversité des modalités d'insertion des immigrés dans la société d'accueil. Ce modèle met également l'accent sur le rôle des migrants eux-mêmes comme agents de leur propre processus d'intégration, alors que des modèles plus globaux suggèrent que les migrants se moulent dans des prototypes définis par la société d'accueil. 
3 La théorie de l'assimilation segmentée est basée sur les différentes performances des groupes d'immigrants afin d'« expliquer ce qui détermine le segment de la société américaine auquel un groupe spécifique d'immigrants pourra s'assimiler » (Zhou, 1999: 201). Zhou décrit trois modèles possibles: l'intégration ascendante vers la classe moyenne; l'intégration descendante vers le sous-prolétariat; et la mobilité économique ascendante combinée au maintien de la culture du groupe. L'intégration des Jamaïcains aux États-Unis se caractérise par une dualité : discrimination raciale à leur égard, d'une part, et niveaux d'éducation et de formation élevés, d'autre part (Portes et Rumbaut, 1996: 255; Rumbaut et Portes, $2001: 305$; Portes et Zhou, 1993 : 92). Rumbaut et Portes écrivent que «l'intégration des originaires des Antilles représente un terrain disputé, dans lequel les pressions vers le bas, qui résultent d'une discrimination externe sont contrebalancées par les ressources intellectuelles et matérielles des familles, et leur engagement à voir leurs enfants s'en sortir ». Les Jamaïcains de Floride du sud peuvent être déchirés entre leurs affinités raciales avec les Afro-Américains, d'une part, et leur désir de maintenir la position sociale qu'ils possédaient à la Jamaïque, de l'autre. Cependant, l'une des critiques de l'assimilation segmentée est qu'elle sous-entend que l'assimilation à la communauté afro-américaine est synonyme d'intégration descendante. Foner (2005) indique qu'au contraire l'identification aux Afro-Américains peut offrir aux Jamaïcains des opportunités de mobilité socio-économique ascendante.

4 Portes et Rumbaut (1996: 250) affirment que, dans le cas des enfants d'immigrants, l'assimilation est segmentée en raison du contexte social dans lequel ils grandissent et des modalités différentes d'acculturation. Cette hypothèse vaut également pour les immigrants de la première génération, qui sont affectés de la même façon par le contexte dans lequel ils sont accueillis, et dont l'insertion est également liée à leur milieu d'origine. Ces auteurs décrivent trois facteurs touchant à l'insertion des enfants d'immigrés dans la société d'accueil. Le premier est la couleur de la peau, qui, selon eux, n'est pas seulement caractéristique de l'immigrant, mais aussi de la société d'accueil, qui a des préjugés de race. Zhou (1999 : 201) considère également que la race est un facteur structurel, la discrimination raciale dans la société d'accueil renforçant l'importance de la race. Le second facteur est le lieu géographique où l'immigrant s'installe - souvent, du moins au début, dans les inner cities, en raison des difficultés économiques auxquelles ils doivent faire face. Le troisième facteur est constitué par les changements sur le marché du travail américain qui, suite à la désindustrialisation, ont provoqué une demande accrue à la fois d'emplois peu qualifiés et peu payés et d'emplois hautement qualifiés, qui exigent une formation plus élevée. S'y ajoute une demande décroissante d'emplois de qualification moyenne dans l'industrie, qui offrent plus de possibilités de mobilité économique ascendante à ceux qui ont peu de formation. Tous ces facteurs valent pour les immigrants jamaïcains en Floride du sud et à Toronto : la plupart des Jamaïcains sont noirs, et ils tendent à s'installer dans des grandes aires métropolitaines. En troisième lieu, ils sont vulnérables aux changements du marché du travail des sociétés dans lesquelles ils vivent, bien que leur niveau d'éducation relativement élevé leur permette de prétendre à une mobilité socioéconomique ascendante. 


\section{Les politiques d'immigration}

5 Les politiques d'immigration aux États-Unis et au Canada ont été en étroite correspondance, alors même que les deux pays ont fréquemment modifié ces politiques durant tout le XXe siècle, en particulier dans les années 1920, en 1952 et dans les années 1960. Ces modifications ont des similitudes, à la fois dans leur évolution et dans leur contenu. Il existe pourtant des différences entre les politiques des deux pays, de même qu'entre les immigrants que reçoit chacun d'eux. La présente recherche sur ces ressemblances et différences se limitera à la période antérieure au 11 septembre 2001, qui marque un tournant des politiques d'immigration aux États-Unis, où les préoccupations de sécurité prennent le pas sur les considérations socio-économiques.

6 La différence la plus évidente est l'utilisation, au Canada, du système à points. Les ÉtatsUnis ne pratiquent pas ce système, mais la politique d'immigration y est dominée par l'accent mis sur le regroupement familial, bien qu'il y ait aussi des catégories spécifiques d'immigrants: travailleurs qualifiés, professions libérales ou hommes d'affaires. Il en sera question plus loin. Une seconde différence importante entre les politiques d'immigration aux États-Unis et au Canada est que les immigrants sanspapiers sont un objet de préoccupation beaucoup plus important aux États-Unis, en raison notamment de leur proximité avec le Mexique et la Caraïbe, et de la frontière commune entre États-Unis et Mexique (Halli et Driedger, 1999 : 25).

\section{La politique canadienne d'immigration}

7 La politique canadienne d'immigration a beaucoup évolué au cours du XXe siècle. Le changement le plus radical est intervenu au cours des années 1960, lorsque la qualification et l'instruction ont remplacé les préférences raciales comme critères d'admission des immigrants. Avant 1962, la politique d'immigration reposait principalement sur l'origine raciale et ethnique, faisant porter la préférence sur les Européens et rejetant les autres. Bien que la discrimination raciale ait été officiellement supprimée en 1962, ce n'est qu'en 1967 que la préférence géographique, qui était aussi implicitement raciale, fut abolie (Hawkins, $1988: 11$ ). Les politiques mises en place en 1962 donnèrent la priorité aux immigrants possédant une qualification ou un niveau d'éducation qui leur assurerait la réussite socio-économique au Canada (Parai, 1975 : 456). De plus, les citoyens canadiens et les résidents permanents eurent la possibilité de parrainer des proches venus de leur pays d'origine. Cette politique de parrainage existait déjà, mais elle fut étendue à tous les groupes raciaux, ethniques et nationaux.

8 On relève trois types de critiques au changement de politique intervenu en 1962. D'abord, et parce que dérivant de politiques jusque là centrées sur l'Europe, le parrainage familial signifiait que l'immigration au Canada continuerait à être dominée par la composante européenne. En second lieu, l'accent mis sur le nombre d'années d'éducation limitait l'immigration d'individus venus de pays moins développés, qui n'avaient pas le même accès aux études que les Européens de l'ouest. En troisième lieu, une trop grande latitude était laissée aux officiers d'immigration pour déterminer le profil de chaque individu (Parai, 1875 : 456-457). Le climat de racisme pendant cette période, et les préférences ethniques et raciales précédentes au Canada ne rendaient pas impossible l'idée que ces officiers d'immigration manquent d'impartialité. 
9 Le changement le plus significatif intervint en 1967, avec l'établissement du système à points, basé sur des critères tels que l'âge, le niveau d'éducation, la connaissance de l'anglais ou du français des candidats à l'immigration, ainsi que la résidence de proches au Canada (Parai, 1975 ; Hawkins, 1988 ; Green et Green, 1995). Le but principal n'était pas forcément de diversifier la population canadienne, mais d'accroître le nombre d'immigrants qualifiés, dont le Canada avait besoin.

Ces changements dans les politiques canadiennes d'immigration signifiaient que les personnes originaires de la Caraïbe voyaient s'accroître leurs chances d'entrer au Canada. En 1951, aucun de ces pays ne figurait parmi les dix principaux pays d'origine des immigrants. À partir de 1973, la Jamaïque et Trinidad ont fait partie des cinq premiers pays d'origine, comme le montrent les tableaux 1 et 2 .

Tableau 1 : Les dix principaux pays d'origine des immigrants au Canada

\begin{tabular}{|l|l|l|l|}
\hline 1951 & 1960 & 1968 & 1973 \\
\hline G. Bretagne & Italie & G. Bretagne & G. Bretagne \\
\hline Allemagne & G. Bretagne & États-Unis & États-Unis \\
\hline \hline Italie & États-Unis & Italie & Hong Kong \\
\hline Pays Bas & Allemagne & Allemagne & Portugal \\
\hline Pologne & Pays Bas & Hong Kong & Jamaïque \\
\hline France & Portugal & France & Inde \\
\hline États-Unis & Grèce & Autriche & Philippines \\
\hline Belgique & France & Grèce & Grèce \\
\hline \hline Yougoslavie & Pologne & Portugal & Italie \\
\hline \hline Danemark & Autriche & Yougoslavie & Trinitad \\
\hline
\end{tabular}

Source : The Immigration Program. Ottawa: Manpower and Immigration, 1974.

Tiré de http://www.ucalgary.ca/applied_history/tutor/canada1946/introframe.html

Tableau 2 : Immigrants au Canada par lieu de naissance et période d'immigration (recensement de 2001)

\begin{tabular}{|l|l|l|l|l||l|l|}
\hline & $\begin{array}{l}\text { Avant } \\
1961\end{array}$ & $1961-1970$ & $1971-1980$ & $1981-1990$ & $1991-2001$ & Total \\
\hline États-Unis & 34805 & 46880 & 62835 & 41965 & 51435 & 237920 \\
\hline Amérique centrale et du sud & 5910 & 17155 & 62925 & 102655 & 116005 & 304650 \\
\hline Caraïbes et Bermudes & 6990 & 42740 & 91475 & 68840 & 84005 & 294050 \\
\hline
\end{tabular}




\begin{tabular}{|l|l|l|l|l|l|l|}
\hline Royaume Uni & 217175 & 160005 & 126030 & 60145 & 42645 & 606000 \\
\hline $\begin{array}{l}\text { Autres pays d'Europe de } \\
\text { l'ouest et du nord }\end{array}$ & 248830 & 86820 & 56345 & 45595 & 57235 & 494825 \\
\hline Europe de l'est & 135425 & 36595 & 30055 & 104825 & 164465 & 471365 \\
\hline Europe du sud & 207900 & 232255 & 126095 & 55620 & 93500 & 715370 \\
\hline Afrique & 4635 & 23830 & 54655 & 59710 & 139770 & 282600 \\
\hline Asie du centre ouest et Moyen- & 4445 & 13360 & 29675 & 75885 & 162220 & 285585 \\
\hline Asie de l'est & 18325 & 36360 & 97610 & 155070 & 423235 & 730600 \\
\hline Asie du sud-est & 2240 & 14095 & 107445 & 159660 & 185665 & 469105 \\
\hline \hline Asie du sud & 3845 & 26600 & 77230 & 101110 & 295110 & 503895 \\
\hline \hline Océanie et autres pays & 3950 & 8870 & 13910 & 10415 & 15380 & 52525 \\
\hline
\end{tabular}

Source : Statistiques du Canada, Recensement de la population, « Population immigrée par lieu de naissance et période d'immigration » tiré de http://www40.statcan.ca/l01/cst01/demo24a.htm

11 En 1966, $88 \%$ des immigrants au Canada venaient d'Europe, mais ce pourcentage se réduisit à $51 \%$ en 1970 . Les personnes originaires des Antilles figurèrent alors parmi les dix principaux groupes d'immigrants (8,3\% en 1970). En fait, depuis 1975, plus d'immigrants provenaient des pays en développement que d'Europe, et le recensement de 1996 a montré que plus de la moitié de la population immigrée n'était pas d'origine européenne (Mitchell, 1997, cité par Halli et Driedger, 1995 : 51). Bien qu'au cours des années récentes d'autres pays aient dépassé la Jamaïque dans l'immigration au Canada, celle-ci continue à faire partie des dix pays les plus représentés à Toronto, comme le montre le tableau 3 ci-après. Ce tableau fait aussi apparaître la plus forte représentation d'autres pays en développement dans cette immigration.

Tableau 3 : Toronto selon les dix principaux pays-sources (demandeurs principaux et personnes rattachées)

\begin{tabular}{|l|l|l|l|l|l|l|l|l|l|}
\hline $\begin{array}{l}\text { Pays 2000 } \\
2001 \\
2002\end{array}$ & N & $\%$ & Rang & N & $\%$ & Rang & N & $\%$ & Rang \\
\hline Inde & 15841 & 14,39 & 2 & 17625 & 14,09 & 2 & 18290 & 16,39 & 1 \\
\hline Chine & 18544 & 16,85 & 1 & 21476 & 17,17 & 1 & 17584 & 15,76 & 2 \\
\hline Pakistan & 10753 & 9,77 & 3 & 11581 & 9,26 & 3 & 10357 & 9,8 & 3 \\
\hline Philippines & 4034 & 3,66 & 5 & 6028 & 4,82 & 4 & 5260 & 4,71 & 4 \\
\hline Iran & 3036 & 2,76 & 7 & 2977 & 2,38 & 8 & 4711 & 4,22 & 5 \\
\hline
\end{tabular}




\begin{tabular}{|l|l|l|l|l|l|l|l|l|l|}
\hline Sri Lanka & 4237 & 3,85 & 4 & 4274 & 3,42 & 6 & 3711 & 3,33 & 6 \\
\hline Émirats arabes unis & 2258 & 2,05 & 8 & 3338 & 2,67 & 7 & 3178 & 2,85 & 7 \\
\hline Corée & 3536 & 3,21 & 6 & 4684 & 3,74 & 5 & 2976 & 2,67 & 8 \\
\hline Ukraine & 2029 & 1,84 & 11 & 2313 & 1,85 & 14 & 2254 & 2,02 & 9 \\
\hline Jamaïque & 2068 & 1,88 & 10 & 2337 & 1,87 & 13 & 2054 & 1,84 & 10 \\
\hline Russie & 2149 & 1,95 & 9 & 2430 & 1,94 & 12 & 2014 & 1,80 & 11 \\
\hline Arabie saoudite & 1464 & 1,33 & 17 & 2603 & 2,08 & 9 & 1793 & 1,61 & 12 \\
\hline Roumanie & 1911 & 1,74 & 13 & 2445 & 1,95 & 10 & 1670 & 1,50 & 15 \\
\hline Total des dix premiers \\
pays & 66456 & 60,37 & & 77031 & 61,58 & & 70375 & 2000 & \\
\hline \hline Total autres pays & 43613 & 39,63 & & 48083 & 38,42 & & 41205 & 36,93 & \\
\hline Total & 110069 & 100,00 & & 125114 & 100,00 & & 111580 & 100,00 & \\
\hline
\end{tabular}

Source : Citoyenneté et immigration Canada, faits et chiffres 2002, vue d'ensemble sur l'immigration tiré de http://www.cic.gc.ca/english/pub/facts2002/toronto/toronto_2.html

12 Un autre changement, moins significatif, et cependant important, encouragea les immigrants des Antilles : ce fut l'inclusion des peuples caribéens dans le Schéma de prêt et d'aide (Assisted Passage Loan Scheme, APLS), qui n'était, au départ, accessible qu'aux immigrants venus d'Europe (Parai, 1975 : 463). L'APLS est un système de prêts qui a aidé les immigrants à payer leur voyage vers le Canada. Il apporta aux personnes originaires des Antilles l'accès aux ressources nécessaires.

13 Le système légal canadien se caractérise par son système à points, base des politiques migratoires. Les immigrants potentiels ont trois principales possibilités d'entrée. La première concerne les travailleurs qualifiés, qui doivent avoir au minimum un an d'expérience dans une profession entrant dans la Classification nationale des emplois. S'ils n'ont pas d'emploi assuré avant de demander à immigrer, ils doivent prouver leur capacité financière à subvenir à leurs besoins et à ceux de leur famille. On dénombre six facteurs de sélection, chacun offrant à l'immigrant de gagner des points, qui peuvent atteindre cent. Le nombre minimum de points requis pour être admis à immigrer est de soixante-sept sur cent. Les six catégories sont : éducation, connaissance de la langue anglaise ou française, expérience de travail, âge, engagement d'emploi au Canada et capacité d'adaptation.

14 Le second mode d'immigration est celui de la création d'une entreprise, avec trois catégories: investisseurs, entrepreneurs, et travailleurs à leur compte. Les investisseurs doivent avoir une expérience dans la direction d'une affaire, ainsi qu'un capital net de $800000 \$ C$, dont ils s'engagent à investir 400000 au Canada. Des entrepreneurs immigrant au Canada, on attend qu'ils contribuent au développement économique en possédant des entreprises et en créant des emplois. Un chiffre d'affaires 
net de $300000 \$ C$ est requis. La troisième catégorie d'immigration d'affaires concerne les personnes travaillant à leur compte, qui ont beaucoup moins de restrictions et d'obligations. En plus des critères correspondant à la catégorie choisie, ils doivent obtenir au minimum trente-cinq des cent points possibles dans les catégories suivantes : éducation, expérience, âge, connaissance de l'anglais ou du français, et adaptabilité.

La troisième catégorie autorise les citoyens canadiens et les résidents permanents de plus de dix-huit ans vivant au Canada à parrainer des membres de leur proche famille ${ }^{1}$. Les parrains doivent être d'accord pour prendre en charge les membres de leur famille pour une période de trois à dix ans, selon l'âge et le degré de parenté. Le parrainage ne requiert aucun des critères additionnels prévus dans le système à points utilisé pour les autres catégories.

Il existe d'autres possibilités d'immigration individuelle au Canada, comme l'adoption, l'immigration provinciale ou le statut de réfugié. Le graphique 1 illustre la proportion d'immigrants au Canada en 2002 dans chacune des catégories énumérées ci-dessus. La majorité est entrée au titre du travail qualifié (53,7 \%), et secondairement au titre du regroupement familial.

\section{Graphique 1 : Arrivées au Canada en 2002 par catégorie d'immigrés (demandeurs principaux et} personnes rattachées)

Légendes du graphique

- Provincial Nominees : Nommés par les provinces

- Refugee : Réfugiés

- Other: Autres

- Family : Famille

- Entrepreneurs

- Self Employed : Travailleurs à leur compte

- Investor : Investisseurs

- Skilled Workers : Travailleurs qualifiés

Total: 229058

Source: Chiffres Data Warehouse Extract Field Operations Support System (FOSS), Septembre 2003, à partir de Citoyenneté et immigration au Canada « Statistiques 2002 du Programme Immigration d'affaires 2002 » tiré de http://www.cic.gc.ca/english/business/bus-stats2002.html.

Il apparaît difficile de faire entrer les immigrants dans un mode d'immigration spécifique, en raison de processus divers et parfois complexes. Quelques-uns des Jamaïcains immigrés à Toronto et interrogés au cours de la présente recherche ont indiqué que, bien qu'entrés au Canada en tant qu'étudiants ou que visiteurs, ils se marièrent plus tard, ce qui leur permit de contourner le processus d'accès au statut de résident permanent. Le tableau 4 fait apparaître les catégories selon lesquelles les Jamaïcains interrogés ont immigré.

Tableau 4 : Demandeurs jamaïcains par catégorie d'admission

\begin{tabular}{|l|l|l|}
\hline Catégorie d'admission & Toronto & Floride du sud \\
\hline Parrainage par parent, enfant ou proche parent & 9 & 27 \\
\hline Parrainage par époux/se & 6 & 9 \\
\hline Parrainage par employeur & 5 & 6 \\
\hline
\end{tabular}




\begin{tabular}{|l|l|l|}
\hline Entré avec un visa d'étudiant & 2 & 6 \\
\hline Demande individuelle après entrée comme visiteur & 5 & 0 \\
\hline Travailleur qualifié & 25 & $\mathrm{n} / \mathrm{a}$ \\
\hline Total & 52 & 48 \\
\hline
\end{tabular}

Source : Entretiens menés par l'auteure.

Dans l'échantillon des Jamaïcains interrogés au Canada, quoique le mode d'entrée prédominant des immigrants soit la famille, il existe quelques recoupements entre ceux qui ont émigré grâce à une relation familiale et ceux qui sont entrés comme travailleurs qualifiés ou travailleurs indépendants. Le plus souvent, les immigrants ont en fait été admis en tant que travailleurs qualifiés, bien que le parent ait entrepris ou aidé au processus d'immigration. Les infirmières sont les plus nombreuses dans la catégorie des travailleurs qualifiés admis, suivies des enseignants.

\section{Politique d'immigration aux États-Unis}

Il existe trois voies d'accès principales à la migration volontaire aux États-Unis, si l'on exclut les réfugiés et demandeurs d'asile. La catégorie la plus fréquemment utilisée, et numériquement la plus nombreuse, est la réunification familiale. Dans cette catégorie, les citoyens américains peuvent parrainer les membres les plus proches de leur famille, qui se répartissent en quatre catégories : les enfants célibataires de citoyens américains et leurs enfants ; les époux et enfants célibataires de résidents légaux permanents ; les enfants mariés de citoyens américains, ainsi que leurs époux et enfants; enfin, les frères et soeurs (y compris leurs époux et enfants) de citoyens américains de plus de 21 $a^{2} s^{2}$. La réunification familiale est le premier mode d'immigration chaque année. Par exemple, pour l'année civile 2000,69\% des immigrants aux États-Unis furent admis sous cette catégorie (Martin, $2004: 53$ ).

La deuxième catégorie, par ordre d'importance, est celle des travailleurs migrants. Dans ce groupe, la préférence est, par ordre décroissant, donnée aux catégories ciaprès : non-résidents possédant une qualification particulièrement recherchée ; nonrésidents ayant des diplômes supérieurs ; travailleurs qualifiés, professions libérales et non qualifiées qui peuvent répondre aux pénuries du marché du travail; professions spécifiques, telles que les professions religieuses; enfin, immigrants qui investissent et créent des possibilités d'emploi pour des citoyens et résidents états-uniens ${ }^{3}$. Ceux qui sont entrés par cette voie représentent avec leur famille $13 \%$ du total des immigrants aux États-Unis pour l'année civile 2000 (Martin, 2004 : 53).

La troisième voie d'obtention de la résidence légale permanente est celle de la «loterie de la diversité ». Le gouvernement des États-Unis admet chaque année 50000 résidents venant de pays qui ont envoyé moins de 50000 immigrants pendant les cinq dernières années. Ce programme est géré par le Centre national des visas du Département d'État ${ }^{4}$. Environ 10 à 15 millions d'étrangers entrent annuellement par le biais de cette loterie (Martin, 2004 : 53). Ceux qui sont retenus peuvent alors demander le statut de résident permanent. 

"la capacité à obtenir un emploi compatible avec la qualification", ce qui est nécessaire pour permettre une adaptation culturelle. L'ajustement professionnel n'est pas seulement le reflet du capital humain de l'immigrant, mais fait aussi apparaître certaines limites de la société d'accueil, en termes de capacité à bien recevoir les immigrants, particulièrement les personnes de couleur. Citant Weber (éd.1968), Neuwirth (1993 : 63) note l'importance de la race lorsqu'elle montre que « la définition par la race... est employée dans la vie de tous les jours comme prétexte pour exclure de la compétition pour les opportunités économiques et sociales des groupes possédant certaines caractéristiques phénotypiques ou somatiques ».

es différences d'insertion des immigrants sur le marché du travail des pays d'accueil sont liées à des facteurs spécifiques aux immigrants et à leur milieu, ainsi qu'à celui de la société d'accueil. Piché et al (1999: 185) affirment que ces variations sont fonction de l'origine nationale, et ils proposent trois types principaux d'explication à ces variations. Le premier est l'éventail des circuits pour différents groupes d'immigrants ; le second est la difficulté que certains groupes rencontrent à transférer leur capital 
humain dans leur nouveau pays, et le troisième est la discrimination à laquelle doivent faire face certains groupes de minorités " particulièrement visibles $»^{5}$.

En théorie, la politique d'immigration canadienne montre que les travailleurs instruits et qualifiés sont les bienvenus dans la société canadienne et qu'il existe une demande de l'expertise et de la diversité culturelle qu'ils apportent. Pourtant, en pratique, il existe des frontières plus importantes qui freinent l'intégration des immigrants non blancs (ou minorités visibles) par rapport aux immigrants blancs (Halli et Driedger, 1999 ; Henry, 1994). En plus de la discrimination raciale à laquelle ils sont confrontés dans leur recherche d'emploi et de mobilité ascendante dans leur profession, les Jamaïcains, comme beaucoup d'autres immigrants de pays en voie de développement, doivent aussi faire face à la non-reconnaissance par les autorités canadiennes de leurs diplômes, références ou expériences à l'étranger. La non-validation de leur capital humain personnel freine leur capacité à réussir dans la société d'accueil (Neuwirth, 1999 : 57). Elle suggère que ce n'est pas le manque d'éducation ou de qualification qui les empêche de trouver des emplois en rapport avec leurs capacités, mais la structure du marché du travail du pays d'accueil, où il peut ne pas y avoir de demande du type de qualification qu'ils possèdent. Les biais structurels de la société d'accueil empêchent aussi certains types d'immigrants d'accéder à des secteurs et des positions plus attractifs.

Neuwirth (1999: 59) montre que les recensements et les statistiques ne rendent pas suffisamment compte des obstacles à l'intégration économique auxquels font face les immigrants non européens au Canada. À partir de sa précédente recherche sur les immigrants d'Asie du sud-est au Canada (Neuwirth, 1993) et de l'étude de OpokuDapaah (1995) sur les réfugiés somalis dans l'agglomération de Toronto pour appuyer son argument, Neuwirth confirme que la discrimination envers les immigrants «visibles » limite leurs perspectives d'emploi. Elle suggère la nécessité de recherches plus développées sur l'exclusion des immigrants non européens d'emplois pour lesquels ils sont qualifiés. Parmi les freins au transfert de qualification de pays tels que la Jamaïque vers le Canada, on note l'expérience nécessaire au Canada et le rejet des attestations délivrées dans la plupart des pays en voie de développement. On donnera ci-après quelques exemples de la perception individuelle de leur marginalisation par des Jamaïcains interrogés au cours de notre recherche. L'échantillon se compose de 52 adultes jamaïcains dans l'agglomération du grand Toronto et de 48 pour l'aire métropolitaine de Miami (y compris Fort Lauderdale), qui tous ont immigré entre 1965 et 1994. Tous ont vécu aux États-Unis ou au Canada depuis dix ans au moins après la date des entretiens.

Les originaires de Jamaïque au Canada interrogés au cours de cette recherche ont exprimé leur frustration face aux obstacles à l'entrée sur le marché du travail. Une des difficultés les plus fréquentes réside dans l'exigence de diplômes canadiens et/ou une expérience au Canada, notamment pour travailler dans le domaine de la santé ou de l'éducation. Selon les interviewés, malgré la pénurie d'enseignants au Canada, les conseils scolaires locaux qui font les recrutements privilégient l'expérience antérieure au Canada et semblent peu intéressés par les candidats originaires de la Jamaïque. Ils évoquent la question de la surqualification, de la difficulté d'obtention d'une promotion et de l'existence d'un racisme subtil dans le monde du travail. La discrimination raciale est aussi présente dans le logement. 
ien que les Jamaïcains en Floride du sud soient confrontés à des problèmes de discrimination similaires dans le travail ainsi qu'à la question de la reconnaissance des diplômes jamaïcains, le contexte de leur réception dans cette région est différent du fait de la nature et des caractéristiques de l'immigration en Floride du sud. La forte présence d'une population née à l'étranger y joue un rôle dans l'intégration des immigrants dans la société locale. Les immigrants jamaïcains obtiennent des résultats relativement bons en matière d'éducation et d'emploi, comme le soulignent Portes et Rumbaut (1996 : 59), lorsqu'ils analysent la réussite des immigrés aux États-Unis. Ainsi, parmi les Jamaïcains, 67,9 \% ont un diplôme de l'enseignement secondaire, une position comparable à celle de la moyenne américaine, d'après le Bureau fédéral du recensement.

En Floride du sud comme à Toronto, les Jamaïcains instruits aux États-Unis ou au Canada ont un avantage sur les marchés du travail de ces deux pays. Malgré les défis auxquels ils sont confrontés, leur statut socio-économique, tel que reflété par leur éducation, leur profession et leurs revenus, est assez élevé. Les chiffres du Recensement montrent que les immigrants jamaïcains aux États-Unis ont un statut socio-économique plus élevé qu'à Toronto, sur la base de l'éducation et des revenus (tableaux 5, 6 et 7). Il est plus difficile de discerner les différences professionnelles entre les deux populations, en raison des divergences entre les catégories des recensements aux ÉtatsUnis et au Canada.

Tableau 5 : Jamaïcains de 15 ans* et plus par niveau d'éducation

\section{(pourcentages)}

\begin{tabular}{|l|l|l|l|l|}
\hline Niveau d'éducation & États-Unis & Canada & Floride du sud & Toronto \\
\hline Pas de diplôme du secondaire & 29,2 & 27 & 29,5 & 28 \\
\hline Diplôme du secondaire sans baccalauréat & 53,7 & 64 & 57,3 & 63,7 \\
\hline Baccalauréat et plus & 17,1 & 9 & 13,2 & 8,3 \\
\hline
\end{tabular}

* Les 25 ans et plus auraient été préférables, mais les chiffres disponibles pour le Canada ne recensaient que les 15 ans et plus.

Source : Échantillons Floride du sud et Toronto : entretiens par l'auteure.

Source : Statistiques Toronto et Canada : Recensement de la population, C0-0851, Profils des groupes-cibles - Population née en Jamaïque, Montréal, 2001.

Source pour la Floride du sud : Bureau américain du Recensement, échantillon à usage public, Floride, $5 \%$, Recensement de la population 2000, Washington, D.C., 2003.

Source pour les États-Unis : Bureau américain du Recensement, PUMS, Floride, 5 \%, Recensement de la population 2000, Washington, D.C., 2003. Échantillon un pour cent, Recensement de la population 2000, Washington, D.C., 2003.

Tableau 6 : Jamaïcains de 16 ans et plus, par type d'emploi (Poucentages)

\begin{tabular}{|l|l|l|l|l|}
\hline Emploi & $\begin{array}{l}\text { Jamaïcains en } \\
\text { Floride du sud }\end{array}$ & $\begin{array}{l}\text { Jamaïcains aux } \\
\text { États-Unis }\end{array}$ & $\begin{array}{l}\text { Jamaïcains à } \\
\text { Toronto }\end{array}$ & $\begin{array}{l}\text { Jamaïcains au } \\
\text { Canada }\end{array}$ \\
\hline
\end{tabular}




\begin{tabular}{|l|l|l|l|l|}
\hline $\begin{array}{l}\text { Direction, professions } \\
\text { libérales et connexes }\end{array}$ & 26,3 & 24,8 & 31,6 & 32 \\
\hline Services & 25,2 & 28,7 & 22 & 22 \\
\hline Vente et bureaux & 26,7 & 25,2 & 17,8 & 18 \\
\hline $\begin{array}{l}\text { Agriculture, pêche et } \\
\text { forêts }\end{array}$ & 0,2 & 0,2 & 0,1 & 0,6 \\
\hline $\begin{array}{l}\text { Construction, mines \& } \\
\text { entretien }\end{array}$ & 10,4 & 9,1 & 4 & 4 \\
\hline $\begin{array}{l}\text { Production, transports et } \\
\text { déplacement d'engins }\end{array}$ & 10,1 & 10,7 & 24,5 & 23,4 \\
\hline Militaires & 0 & 1,0 & 8 & 8 \\
\hline Sans emploi & 1,0 & 0,2 & 0 & 0 \\
\hline
\end{tabular}

Source pour la Floride du sud : Bureau américain du recensement, PUMS, Floride, $5 \%$, Recensement de la population 2000, Washington, D.C., 2003.

Source pour les États-Unis : Bureau américain du recensement, PUMS, 1 \%, Recensement de la population 2000.

Source pour Toronto et le Canada : Statistiques du Canada, recensement de la population, C0-0851, Profils des groupes-cibles - Population née en Jamaïque, Montréal, 2001.

Tableau 7 : Revenu personnel moyen des Jamaïcains de 15 ans et plus

\begin{tabular}{|l|l|}
\hline Population & Revenus moyens (U.S. Dollars) \\
\hline États-Unis & 27484 \\
\hline Canada & 19231 \\
\hline Floride du sud & 25675 \\
\hline Toronto & 23009 \\
\hline Jamaïcains en Floride du sud & 21651 \\
\hline Jamaïcains à Toronto & 19170 \\
\hline Jamaïcains aux États-Unis & 24742 \\
\hline
\end{tabular}


Source for U.S. : Source pour les États-Unis : Bureau américain du recensement, échantillon un pour cent à usage public (PUMS), Recensement de la population 2000, Washington, D.C., 2003.

Source pour le Canada : Statistiques du Canada, recensement de 1996, « Sélection de statistiques de revenus pour les individus, les familles et les foyers, par zones métropolitaines de recensement », tiré de http://iiiprxy.library.miami.edu:5342/english/Pgdb/famil61f.htm

Source pour les Jamaïcains au Canada : Statistiques du Canada, Profils des groupes- cibles -

Population née en Jamaïque.

\section{Travailler à son compte}

La propension à travailler à son compte est un facteur déterminant de la mobilité socioéconomique des immigrants, car ainsi ils gagnent souvent plus que les salariés (Portes et Rumbaut, 1996: 71; Lofstrom, $2002: 84$ ). Bien que les immigrants Jamaïcains aux États-Unis aient des taux d'emploi à leur compte inférieurs à la moyenne américaine, il y a quelques différences entre les situations aux États-Unis et au Canada qui méritent discussion.

Portes et Rumbaut (1996: 76) énumèrent différentes théories qui tentent d'expliquer pourquoi certains groupes ethniques ont une propension supérieure à d'autres à travailler à leur compte. L'une de ces théories soutient que certains migrants ont plus que d'autres l'esprit d'entreprise pour des raisons culturelles. Mais cette théorie n'explique pas pourquoi des immigrants d'un large éventail de milieux ethniques, culturels et religieux ont tendance à entreprendre, alors que d'autres, de milieux semblables, diffèrent à cet égard.

Selon une autre théorie, les immigrants qui ont l'intention de retourner dans leur pays ont davantage tendance à travailler à leur compte dans l'espoir de constituer un capital qui le leur permette. Portes et Rumbaut rejettent également cette théorie, qui n'explique pas l'esprit d'entreprise dans des groupes comme celui des Cubains, arrivés aux États-Unis avec un très faible espoir de retour. Par contraste, les Mexicains ont plus de chance de retourner chez eux, et pourtant l'auto-emploi et l'entreprenariat y sont moins répandus que chez les Cubains.

Une troisième explication considère que les membres des minorités confrontées à une discrimination sur le marché du travail sont davantage amenés à travailler à leur compte. Henry (1994) soutient cette théorie, et affirme que, parmi les immigrants caribéens à Toronto, « une des principales motivations au travail à son compte était le racisme dans le travail ». Sa recherche identifie les immigrés de la Caraïbe qui, en réponse au racisme au travail, ont monté leur propre entreprise (Henry, 1994: 107-109). Pourtant, cette théorie n'explique pas les différences entre des niveaux d'entreprenariat plus élevés parmi les immigrants chinois et japonais, d'une part, et plus faibles parmi les Philippins et les Jamaïcains, de l'autre. Les uns et les autres sont considérés comme des minorités, et donc susceptibles de discrimination (Portes et Rumbaut, 1996 : 77).

Bien que Portes présente et critique clairement ces théories dominantes qui tentent d'expliquer la plus grande propension de certains groupes à créer leur propre entreprise, il néglige de proposer une alternative. La comparaison d'un même groupe national selon l'espace géographique où il se situe contribue à identifier une autre alternative, plausible quoique incomplète. La société d'accueil a la capacité de créer les 
conditions nécessaires à la réussite des efforts de création d'entreprise. Mais cette explication peut suggérer à tort que tous les immigrants dans une société qui offre les conditions idéales à l'entrepreneur pourraient non seulement saisir les opportunités, mais aussi réussir. Une explication complémentaire nuançant cette vision indique que l'éducation, la qualification ou les ressources que les immigrants apportent avec eux suscitent davantage l'esprit d'initiative lorsqu'elles sont utilisées dans une société qui facilite l'intégration des immigrés. À petite échelle, ce peut être le cas des Jamaïcains en Floride du sud. La combinaison de niveaux élevés de qualification et d'éducation avec leur présence dans une société où le capital social est hautement développé parmi les immigrés facilite un développement plus important de l'entreprenariat.

Par contraste, la population jamaïcaine de Toronto reste entravée par les perceptions discriminatoires, une société qui continue à attendre peu de choses des immigrants des Antilles, et qui ne soutient pas activement leur esprit d'entreprise. La réticence des organismes de crédit à accorder des prêts aux Caribéens a été également citée comme un frein au développement de l'entreprenariat au Canada (Henry, 1994: 114). La dispersion résidentielle de la communauté jamaïcaine joue aussi en faveur de cette situation, et freine la création de davantage d'entreprises jamaïcaines.

\section{Revenus et discrimination des minorités}

Les chercheurs ont également montré que les minorités visibles, dont font partie la plupart des immigrants jamaïcains, tendent à gagner moins que les minorités non visibles. À l'aide de courbes de régression, les premiers montrent que « les immigrants mâles des minorités non visibles ont un avantage de revenus de $30 \%$ sur leurs équivalents de minorités visibles ». De même, "les immigrantes de minorités non visibles ont un avantage de $8 \%$ sur celles des minorités visibles", bien que leurs emplois soient comparables (Basavarajappa et Jones, 1999: 255-257). Ils ont observé que, lorsque des minorités visibles et non visibles du même sexe et d'un âge, d'une éducation et d'une classe sociale comparables travaillent dans le même secteur pour la même durée, les minorités visibles gagnent moins. Ils en ont conclu que la discrimination est une réalité sur le marché du travail canadien, se traduisant par des différences de revenus. De même, Henry (1994: 118) écrit que "en dépit de niveaux d'éducation plus élevés, les membres de minorités visibles étaient moins payés que les autres Canadiens ».

Alors que quelques chercheurs (Basavarajappa et Jones, 1999) affirment qu'il existe des discriminations à l'encontre des minorités visibles, il est également vrai que, dans certains cas, les immigrés jamaïcains gagnent plus que d'autres groupes ethniques. Par exemple, en Floride du sud, les Jamaïcains ont un revenu médian individuel de 17200 \$ US, alors que les Afro-Américains et les Latino-américains ont un revenu médian de 12000 \$. Les Blancs non hispaniques ont un revenu médian de 22800 \$. Les actifs d'origine jamaïcaine expriment leurs difficultés à entrer sur le marché du travail, notamment parce que leurs attestations jamaïcaines ne sont pas prises en compte. Cependant, au cours des années, et parfois après un retour aux études pour accroître le niveau de formation et d'instruction, beaucoup ont pu trouver un emploi dans leur domaine. À la fin, le fait qu'ils soient satisfaits de leur carrière ne réfute pas les résultats de Basavarajappa et Jones qui montrent que les minorités visibles sont souspayées en raison de discriminations. Bien qu'une telle analyse dépasse le cadre du 
présent papier, il ressort des entretiens qu'il existe des a priori qui discréditent l'éducation et l'expérience des immigrants jamaïcains.

\section{Utilité de la discrimination positive et équité dans l'emploi}

discriminations dans l'emploi en mettant ces pratiques hors la loi, mais aussi d'augmenter la représentation des femmes et des minorités sur le marché du travail (Holzer et Neumark, 2000 : 483). Il s'agit d'un concept difficile à mettre en évidence parce qu'il n'y a pas de politique nationale cohérente sur ce thème aux États-Unis. Mettant l'accent sur la difficulté à donner des contours précis aux politiques de discrimination positive, les auteurs les décrivent comme «un amalgame de composantes empruntées à d'autres législations et règlements judiciaires ». Ils relèvent aussi la difficulté à préciser les limites de la discrimination positive, car elle fonctionne à différents niveaux : privé, public, fédéral ou de chaque État fédéré. Alors qu'il n'existe aucune politique fédérale dans le domaine de l'admission à l'université, beaucoup d'institutions d'éducation s'efforcent de diversifier l'origine de leurs étudiants (Holzer et Neumark, $2000: 488$ ).

41 d'accroître le nombre de représentants des minorités, en particulier sur le marché du travail, et aussi dans le domaine de l'éducation. L'immense majorité des Jamaïcains interrogés en Floride du sud (94\%) est favorable à la discrimination positive, ressent son impact positif sur les Jamaïcains, et indique sa race sur les formulaires pour l'emploi ou l'éducation. En dépit de ce soutien massif, le commentaire ci-après d'un étudiant jamaïcain vivant à Miami est typique des déclarations de plusieurs interviewés, en réponse à des questions sur leur perception de l'impact de la discrimination positive sur les Jamaïcains :

«Elle peut sûrement être positive, mais je ne sais pas s'ils s'en servent. Je pense que, en arrivant de la Jamaïque, leur fierté peut faire obstacle à l'utilisation de cet avantage, pourtant très utile ».

En 1986, le gouvernement canadien mit en place plusieurs dispositifs d'égalité des chances, dont le but était d'accroître la présence sur le marché du travail des femmes, des peuples premiers ${ }^{6}$ et des personnes handicapées (Henry, 1994:117; Abu-Laban et Gabriel, 2003 : 131). Le dispositif vise les grandes entreprises « dont les activités sont régulées au niveau fédéral ». Pourtant, ces politiques sont de plus en plus remplacées par l'engagement volontaire à la diversité de la part des entrepreneurs, ce qui est en phase avec les idéaux néolibéraux sur la diminution du rôle du gouvernement dans le secteur privé. Bien qu'elles aient été contestées depuis leur mise en place, ces politiques n'ont, semble-t-il, pas eu beaucoup d'effet sur l'accroissement de la représentation des Noirs dans un large éventail de secteurs (Henry, 1994). En dépit de ces résultats décevants, $79 \%$ de Jamaïcains interrogés à Toronto disent qu'ils sont d'accord avec le dispositif d'égalité des chances dans le domaine de l'emploi, et qu'il est profitable aux Jamaïcains. Très peu d'entre eux mentionnent leur race ou leur ethnie dans les formulaires de demande d'emploi, parce que les entreprises privées ne demandent généralement pas ce type d'information. Ils approuvent ce programme pour différentes raisons :

"Oui, à cause de tous les préjugés dans ce pays, nous en avons besoin. Il y a tant de Noirs qualifiés ici qui ne trouvent pas d'emploi. Je ne parle pas de moi. Je n'ai pas d'instruction, 
cela m'est donc égal de travailler dans une usine, mais mes enfants ont grandi ici, et sont allés dans les mêmes écoles que les autres, et les autres trouvent du travail ici ». (ouvrier d'usine)

"Cela peut être positif pour des gens peu qualifiés, mais pour ceux qui ont une qualification, comme les infirmières, une fois que vous êtres diplômé, c'est plus dur pour eux de leur refuser un emploi à cause de leur race ». (infirmière)

D'autres comme le comptable cité ci-dessous considèrent que le modèle américain de discrimination positive a été positif pour les Noirs aux États-Unis, et devrait peut-être être repris au Canada :

«Oui, c'est la raison pour laquelle les Noirs font leur chemin aux États-Unis. Il y a aussi du racisme partout, mais au moins, ils s'en occupent. Ici, tout est caché, et donc vous ne pouvez rien faire contre. Là-bas, il y a tant de Noirs dans des positions de pouvoir dans les affaires, au gouvernement, dans tous les domaines. Le talent est là, la discrimination positive leur donne les opportunités dont ils ont besoin. Pourquoi ne voyez-vous pas autant de jeunes Noirs instruits et qui réussissent ici? Nous avons besoin de quelque chose de ce genre au Canada».

Surtout, la plupart des Jamaïcains interrogés sont d'accord avec l'équité dans l'emploi ou la discrimination positive. Pourtant, en Floride du sud, leur soutien à ces politiques est plus enthousiaste. Elle est peut-être fonction de l'histoire des politiques dans les deux pays. Historiquement, racisme et discrimination ont été plus patents aux ÉtatsUnis. D'autre part, les Jamaïcains conservent certaines de leurs spécificités, même après immersion dans la société nord-américaine et l'exposition au racisme qui fait parfois surface. Dans les deux pays, les Jamaïcains, surtout lorsqu'ils ont émigré étant adultes, ont exprimé un sentiment de fierté et rejettent les mérites de ces politiques, comme le montre l'exemple de ce dentiste jamaïcano-canadien retraité :

"[Ces politiques] sont peut-être nécessaires aux États-Unis, mais particulièrement nous, les

Caribéens, nous pouvons nous prendre nous-mêmes en charge ".

\section{Conclusion : l'insertion des Jamaïcains dans les sociétés canadienne et américaine}

Ce papier décrit plusieurs traits des sociétés d'accueil et leur contexte affectant l'intégration des Jamaïcains dans les sociétés de Floride du sud et de Toronto. La littérature et les données agrégées présentées ci-dessus montrent combien le contexte d'accueil est vital pour l'insertion des immigrants. Plus spécifiquement, les politiques d'immigration, l'accès au marché du travail, les modèles d'acculturation et d'intégration et le poids démographique des groupes immigrés jouent un rôle non seulement dans le processus migratoire initial, mais aussi dans l'intégration, lorsque les immigrés deviennent un élément des sociétés américaine ou canadienne.

Bien que les politiques d'immigration américaine et canadienne diffèrent, les catégories via lesquelles les immigrants entrent aux États-Unis et au Canada sont semblables. Près de la moitié des Jamaïcains interrogés à Toronto $(48 \%)$ ont indiqué qu'ils avaient immigré grâce au système à points comme travailleurs qualifiés, et pourtant, $73 \%$ ont précisé qu'ils avaient des membres de la proche famille vivant déjà au Canada lorsqu'ils ont immigré. Cette remarque montre que les catégories ne sont pas mutuellement exclusives, les immigrants recevant de l'aide de membres de leur famille, même s'ils immigrent dans des catégories autres que le regroupement familial. En dépit de l'insistance des politiques canadiennes d'immigration sur l'employabilité, il ne faut pas 
en déduire que Toronto attire plus de travailleurs jamaïcains plus instruits ou plus qualifiés que la Floride du sud. Le niveau d'instruction des Jamaïcains est plus élevé en Floride qu'à Toronto, alors qu'un nombre comparable de Jamaïcains dans les deux régions avait de la famille dans le pays d'accueil au moment de l'immigration (respectivement $85 \%$ en Floride et $73 \%$ à Toronto). Par conséquent, la politique d'immigration en Floride du sud comme à Toronto sert à faciliter l'immigration légale, davantage qu'à déterminer les caractéristiques des immigrants.

À partir des réponses des interviewés et de la similarité de leurs profils professionnels, ils semblent également avoir un accès comparable au marché du travail. Pourtant, malgré leur même désir de rester de façon permanente dans le pays d'accueil, davantage de Jamaïcains interrogés en Floride du sud travaillaient à leur compte qu'à Toronto. Les modèles généraux d'acculturation et d'intégration sont proches pour les deux groupes de Jamaïcains. Cependant, aux États-Unis les Jamaïcains ont plus de possibilités de choix d'assimilation, notamment à un groupe spécifique d'Américains : soit le groupe dit dominant (mainstream), soit ceux de la classe moyenne ou ouvrière afro-américaine.

Portes et Rumbaut (1996: 84) décrivent les trois « principaux contextes d'accueil » des immigrants. Il s'agit «des politiques du gouvernement d'accueil, des conditions du marché du travail d'accueil et des caractéristiques de leurs propres communautés d'origine ». Ces trois conditions sont certes importantes, mais ils en négligent une quatrième, également importante : la composition ethnique de la société d'accueil. L'environnement le plus immédiat est celui de leur propre communauté ethnique. Mais d'autres groupes ethniques influent sur leur insertion dans la société en général, comme l'a montré Foner $(1983 ;$ 1985). Institutions et organisations d'immigrés promeuvent l'épanouissement et l'avancement des immigrés noirs dans un contexte de domination raciale.

\section{BIBLIOGRAPHIE}

ABU-LABAN Yasmeen and GABRIEL Christina (2002) Selling Diversity: Immigration, Multiculturalism, Employment Equity, and Globalization, Peterborough, ON, Broadview Press.

BASAVARAJAPPA K.G. and JONES Frank (1999) Visible Minority Income Differences, in Halli, Shiva S. and Leo Driedger, Eds., Immigrant Canada: Demographic, Economic, and Social Challenges, Toronto, University of Toronto Press.

BOOKMAN Milica Z. (2002) Ethnic Groups in Motion: Ethnic Competition and Migration in Multiethnic States, London, Frank Cass Publishers.

CHISWICK Barry R., Ed. (1992) Immigration, Language, and Ethnicity: Canada and the United States, Washington, DC, American Enterprise Institute.

DAVIS Carla P. (2004) Beyond Miami: The Ethnic Enclave and Personal Income in Various Cuban Communities in the United States, International Migration Review, 38 (2). 
DAWKINS Freda (1988) Canada and Immigration: Public Policy and Public Concern, Kingston and Montreal, McGill-Queen's University Press.

DUNN Marvin (1997) Black Miami in the Twentieth Century, Gainesville, University Press of Florida.

FONER Nancy (2005) In a New Land: A Comparative View of Immigration, New York, New York University Press.

FONER Nancy (1985) Race and Color: Jamaican Migrants in London and New York City,International Migration Review, 19 (4).

FONER Nancy (1983) Jamaican Migrants: A Comparative Analysis of the New York and London Experience, New York, New York University, Faculty of Arts and Science, Center for Latin American and Caribbean Studies.

GREEN Alan G. and GREEN David A. (1995) Canadian Immigration Policy: The Effectiveness of the Point System and Other Instruments, The Canadian Journal of Economics, 28 (4b).

GRENIER Guillermo J. and STEPICK III Alex, Eds. (1992) Miami Now! Immigration, Ethnicity, and Social Change, Gainesville, University Press of Florida.

HALLI Shiva S. and DRIEDGER Leo, Eds. (1999) Immigrant Canada: Demographic, Economic, and Social Challenges, Toronto, University of Toronto Press.

HAMILL Pete (2004) The Alloy of New York, in Tamar Jacoby, Éd., Reinventing the Melting Pot: The New Immigrants and What it Means to be American, New York, Basic Books.

HENRY Frances (1994) The Caribbean Diaspora in Toronto: Learning to Live with Racism. Toronto, University of Toronto Press.

HAWKINS Freda (1988) Canada and Immigration: Public Policy and Public Concern. Second Edition. Kingston and Montreal: McGill-Queen's University Press.

HOLZER Harry and NEUMARK David (2000) Assessing Affirmative Action, Journal of Economic Literature, 38 (3).

LOFSTROM Magnus (2002) Labor Market Assimilation and the Self-employment Decision of Immigrant Entrepreneurs, Journal of Population Economics, 15 (1).

MARTIN Philip L. (2004) The United States: The Continuing Immigration Debate, in Wayne A. Cornelius, Takeyuki Tsuda, Philip L. Martin, and James F. Hollifield, Eds.,Controlling Immigration: A Global Perspective. Second Edition. Stanford, Stanford University Press.

McHUGH Kevin E., MIYARES Ines M. and SKOP Emily H. (1997) The Magnetism of Miami: Segmented Paths in Cuban Migration, The Geographical Review, 87 (4).

McWHORTER John (1989) Getting Over Identity, in Tamar Jacoby, Ed., Reinventing the Melting Pot: The New Immigrants and What it Means to be American. New York, Basic Books.

MITCHELL Christopher (1989) International Migration, International Relations, and Foreign Policy, International Migration Review, 23 (3).

MITCHELL Katharyne (1997) Transnational Discourse: Bringing Geography Back In, Antipode, 29 (2).

NEUWIRTH Gertrud (1999) Toward a Theory of Immigrant Integration, in Halli, Shiva S. and Leo Driedger, Eds., Immigrant Canada: Demographic, Economic, and Social Challenges, Toronto, University of Toronto Press. 
NEUWIRTH Gertrud (1993) The Marginalization of South East Asian Refugees, in Vaughan Robinson, Ed., The International Refugee Crisis: British and Canadian Responses, Basingstoke, The MacMillan Press, University of Oxford.

OPOKU-DAPAAH Edward (1995) Somali Refugees in Toronto: A Profile, Toronto, York Lanes Press. PARAI Louis (1975) Canada's Immigration Policy, 1962-74, International Migration Review, 9 (4).

PASTOR Robert (1987) The Impact of U.S. Immigration Policy on Caribbean Emigration: Does it Matter? in Barry B. Levine, Ed., The Caribbean Exodus, New York, Praeger.

PICHÉ Victor, RENAUD Jean and GINGRAS Lucie (1999) Comparative Immigrant Economic Integration, in Shiva S. Halli and Leo Driedger, Eds. Immigrant Canada: Demographic, Economic, and Social Challenges, Toronto, University of Toronto Press.

PORTES Alejandro and RUMBAUT Ruben G. (1996) Immigrant America: A Portrait. Second Edition, Berkeley, University of California Press.

PORTES Alejandro and STEPICK Alex (1993) City on the Edge: The Transformation of Miami, Berkeley, University of California Press.

PORTES Alejandro and ZHOU Min (1993) The New Second Generation: Segmented Assimilation and Its Variants Among Post-1965 Immigrant Youth, Annals of the American Academy of Political and Social Sciences, 530.

ROGERS Reuel (2006) Afro-Caribbean Immigrants and the Politics of Incorporation: Ethnicity, Exception, or Exit, Cambridge, Cambridge University Press.

RUMBAUT Rubén G. and PORTES Alejandro, Eds. (2001) Ethnicities: Children of Immigrants in America, New York, Russell Sage Foundation.

STEPICK Alex, GRENIER Guillermo, CASTRO Max and DUNN Marvin (2003) This Land Is Our Land: Immigrants and Power in Miami, Los Angeles, University of California Press.

TORCZYNE James L. (2003) The Shaping of Toronto's Black Identity: A Demographic Analysis of the Black Community in Toronto and Regions, Montreal, McGill Consortium for Ethnicity and Strategic Social Planning.

VICKERMAN Milton (1999) Crosscurrents: West Indian Immigrants and Race, New York, Oxford University Press.

WATERS Mary C. (1999) Black Identities: West Indian Immigrant Dreams and American Realities, New York, Russell Sage Foundation.

WATERS Mary C. (1994) Ethnic and Racial Identities of Second-Generation Black Immigrants in New York City, International Migration Review, 28 (4).

WEBER Max (édition de 1968) Economy and Society: An Outline of Interpretive Sociology,Vol I and II, Guenther Roth and Claus Wittich, Eds. New York, Bedminster Press.

WILSON Kenneth L. and PORTES Alejandro (1980) Immigrant Enclaves: An Analysis of the Labor Market Experiences of Cubans in Miami, The American Journal of Sociology, 86 (2).

WOOD Charles H. and McCOY Terry L. (1985) Migration, Remittances, and Development: A Study of Caribbean Cane Cutters in Florida, International Migration Review, 19 (2).

ZHOU Min (1999) Segmented Assimilation: Issues, Controversies, and Recent Research on the New Second Generation, in Charles Hirschman, Philip Kasinitz and Josh DeWind, Éds. The Handbook of International Migration: The American Experience, New York, Russell Sage Foundation. 


\section{NOTES}

1. Conjoints, parents, grands-parents, enfants à charge, de même que les frères et sœurs, cousins, cousines et petits-enfants non mariés et orphelins de moins de dix-huit ans.

2. Source: Département américain de la Sécurité intérieure, Annuaire 2002 des statistiques de l'immigration, octobre 2003.

3. Source : USCIS, à partir de http://uscis.gov/graphics/howdoi/immstatemp.htm 3/14/05.

4. USCIS, à partir de http://uscis.gov/graphics/howdoi/divlott.htm 3/14/05.

5. Le terme « minorité visible » est utilisé au Canada en référence à la population visiblement non blanche ou non européenne.

6. First Nations est le terme habituel pour faire référence aux peuples originaires d'Amérique, qui remplaça le mot «Indiens" au cours des années 1970. Pour plus de détails, voir http:// www.aidp.bc.ca/terminology_of_native_aboriginal_metis.pdf.

INDEX

Index géographique : Floride - Miami, Ontario - Toronto

Mots-clés : intégration sociale

\section{AUTEURS}

\section{TERRY-ANN JONES}

Ph. D., Fairfield University, Department of Sociology and Anthropology, 1073 North Benson Road, Fairfield, CT 06824-5195. 